\title{
NIR Follow-Up of the VVDS 02hr Field: First Results
}

\author{
S. Temporin ${ }^{1}$, A. Iovino ${ }^{1}$, H. J. McCracken ${ }^{2,5}$, M. Bolzonella ${ }^{3}$, \\ M. Scodeggio ${ }^{4}$, and the VVDS Team $\dagger$ \\ ${ }^{1}$ INAF-Osservatorio Astronomico di Brera, Via Brera 28, 20121 Milano, Italy \\ ${ }^{2}$ Institut d'Astrophysique de Paris, UMR 7095, 98 bis Bvd Arago, 75014 Paris, France \\ ${ }^{3}$ INAF-Osservatorio Astronomico di Bologna, Via Ranzani 1, Bologna, Italy \\ ${ }^{4}$ INAF-IASF, Via Bassini 11, Milano, Italy \\ ${ }^{5}$ Observatoire de Paris, LERMA, 61 Avenue de l'Observatoire, 75014 Paris, France
}

\begin{abstract}
We present a new K-band survey covering $623 \operatorname{arcmin}^{2}$ in the VVDS 0226-0430 deep field down to a limiting magnitude $\mathrm{K}_{\text {Vega }} \leqslant 20.5$. We use the spectroscopic sample extracted from this new K-band catalogue to assess the effectiveness of optical-near infrared color selections in identifying extreme classes of objects at high redshift.
\end{abstract}

Keywords. Infrared: galaxies, surveys, galaxies: evolution

\section{The VVDS F02 K-selected sample}

Near-infrared (NIR) selected samples are ideal tools for studying the process of mass assembly at intermediate/high redshift, thanks to the advantages of NIR with respect to optical selection. Within the context of the VIMOS-VLT Deep Survey (see Garilli et al., Lamareille et al., and Vergani et al., this volume, for additional information on the VVDS), we present a new K-band survey in the VVDS 0226-0430 deep field (F02), already covered by the purely flux-limited VVDS spectroscopic survey $\left(17.5 \leqslant \mathrm{I}_{\mathrm{AB}} \leqslant 24\right.$; Le Févre et al. 2005). Deep ancillary photometric data in this field are available through the VVDS (BVRI; McCracken et al. 2003) and the CFHT Legacy Survey $\left(u^{*} g^{\prime} r^{\prime} i^{\prime} z^{\prime}\right)$. The new K-band data, obtained with SOFI at ESO-NTT, extend those described in Iovino et al. (2005), thus covering a total contiguous area of $623 \mathrm{arcmin}^{2}$ to a limiting magnitude $\mathrm{K}_{\text {Vega }} \leqslant 20.5$ (90\% completeness). Our photometric catalogue includes 8857 objects down to $\mathrm{K}_{\text {Vega }} \leqslant 20.25$. Galaxy counts are in good agreement with those from the literature. The angular correlation function does not show any peculiarity as a function of magnitude and angular scale and is broadly in agreement with results from the literature. The K-selected spectroscopic sample contains 1792 galaxies with good quality redshifts. A minimal incompleteness in color arises at $\mathrm{K}_{V e g a}>19.8$, as the reddest objects are disfavoured by the $\mathrm{I}_{\mathrm{AB}} \leqslant 24$ limit. For this red tail of the galaxy color distribution we rely on the wide multiwavelength coverage to obtain good quality photometric redshifts following the method adopted in Ilbert et al. (2006). For this purpose, we note that the use of K-band data reduces considerably the percentage of catastrophic errors (see Appendix). A detailed description of this K-band sample is given in Temporin et al. (2007a). Since the VVDS survey is purely flux-limited, this sample is ideal for assessing the effectiveness of different optical-NIR color selections to identify extreme classes of objects like extremely red objects (EROs) and high-redshift galaxies (BzK; Daddi et al. 2004).

$\dagger$ The complete author list, as well as bibliographic references can be found in the Appendix. 

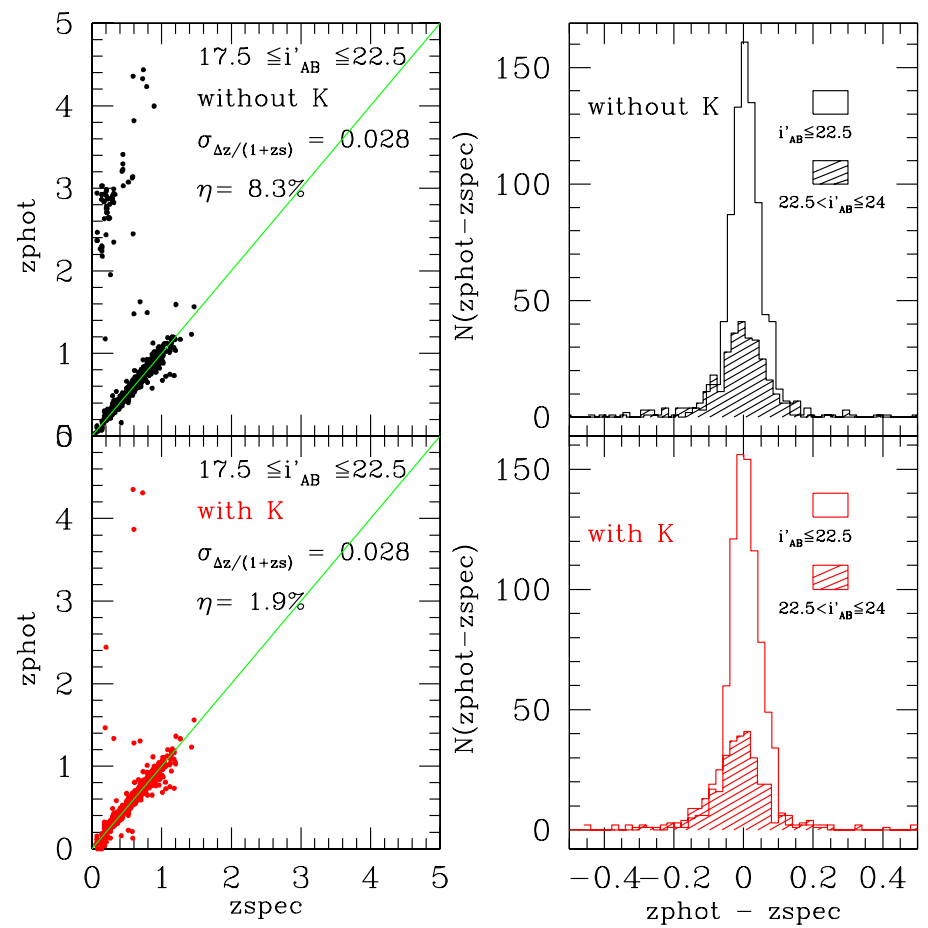

Figure 1. Comparison between spectroscopic and photometric redshifts obtained with and without the use of the K-band. Distributions of the difference between photometric and spectroscopic redshifts are shown for two magnitude ranges.

Some of our first results are summarized in the Appendix, while a thorough discussion of the various color selections is presented in Temporin et al. (2007b).

\section{Appendix A. On-line material}

\section{A.1. Photometric redshifts}

Photometric redshifts have been derived for the whole K-band catalogue by using the code Le Phare (developed by S. Arnouts and O. Ilbert; http://www.lam.oamp.fr/arnouts/LE_ PHARE.html), following the method described in Ilbert et al. (2006). The use of photometric redshifts in our case is important especially for the red tail of the galaxy color distribution, which is missed by the spectroscopic survey because of the imposed magnitude limit $\left(\mathrm{I}_{\mathrm{AB}} \leqslant 24\right)$. For these objects the redshift distribution of the spectroscopic sample cannot be introduced as an a priori information in the probability distribution function. In this case, the quality of the photometric redshifts is significantly improved by the additional information coming from the K-band data. In Fig. 1 we show a comparison between spectroscopic redshifts and photometric redshifts obtained with and without the use of the K-band data. It appears clearly that the use of the K-band reduces considerably the fraction of catastrophic errors $\eta$. Further details are given in Temporin et al. (2007a). 

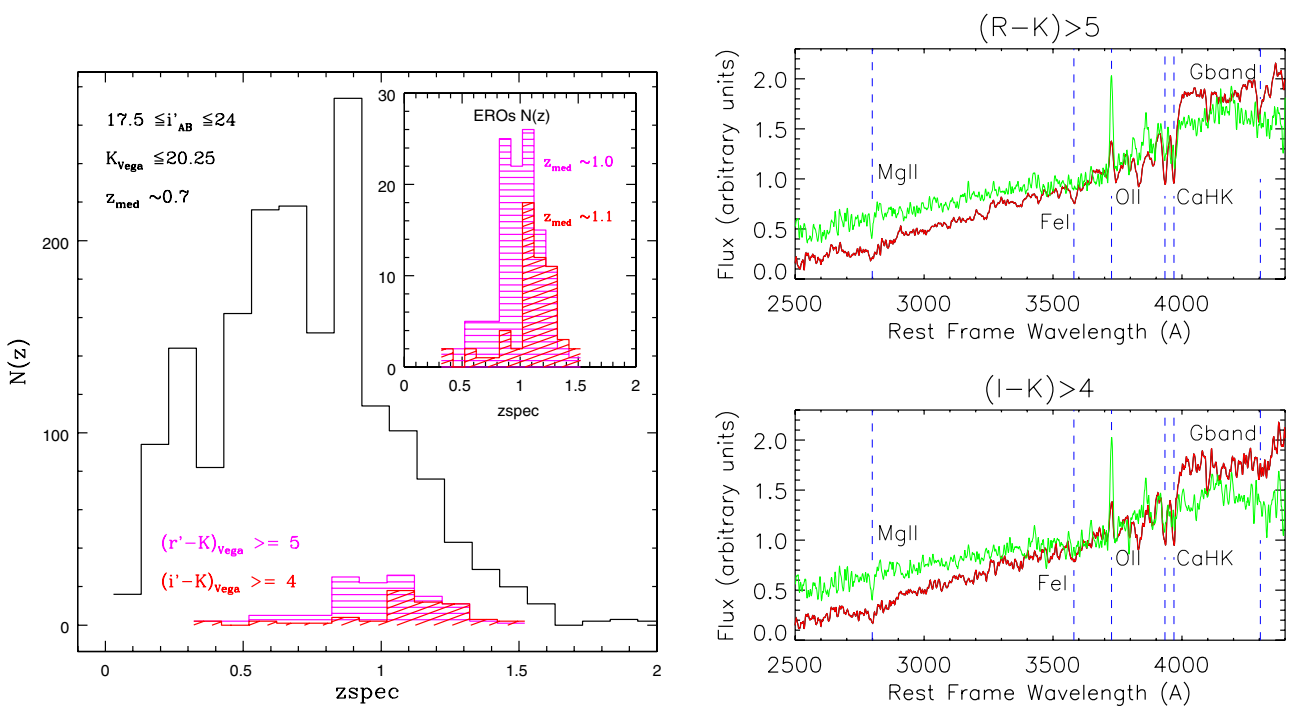

Figure 2. Left. Redshift distributions of the whole spectroscopic sample (black), the $\left(\mathrm{r}^{\prime}-\mathrm{K}\right)$-EROs sample (magenta), and the (i'-K)-EROs sample (red). Right. Composite spectra of the "early"-type (red) and "late"-type (green) galaxies extracted from the two EROs samples.

\section{A.2. EROs and high redshift objects in the K-selected spectroscopic sample: Color selections}

Within our K-selected spectroscopic sample we have identified EROs according to two color selections, $\left(\mathrm{r}^{\prime}-\mathrm{K}\right)_{\text {Vega }}>5$ and $\left(\mathrm{i}^{\prime}-\mathrm{K}\right)_{\text {Vega }}>4$. Our spectroscopic sample includes 148 and 70 EROs with secure redshifts that satisfy the 1st and 2nd criterion, respectively, down to $K_{\text {Vega }} \leqslant 20.25$. The whole spectroscopic sample has a median redshift $z_{\text {med }} \sim$ 0.7, while EROs have $\mathrm{z}_{\text {med }} \sim 1.0-1.1$ (Fig. 2), in agreement with other samples from the literature. The color $\left(\mathrm{i}^{\prime}-\mathrm{K}\right)$ appears more effective than $\left(\mathrm{r}^{\prime}-\mathrm{K}\right)$ in selecting galaxies within a narrow redshift range, up to $\mathrm{z} \sim 1.4$.

A first comparison of the rest-frame observed spectral energy distributions (SEDs) with Coleman, Wu, and Weedman (1980) templates suggests that both samples of EROs are dominated by early-type galaxies. This is confirmed by the best-fit models resulting from the SED fitting with the code Le Phare. Spectra of both samples, accordingly divided into two broad classes (early and late), were used to build the composite spectra. The "early"-type composite spectra built from 96 ( $\left.\mathrm{r}^{\prime}-\mathrm{K}\right)$-EROs and 54 (i'-K)-EROs, show spectral features typical of early-type galaxies. The "late"-type composite spectra built from 17 ( $\left.\mathrm{r}^{\prime}-\mathrm{K}\right)$-EROs and 15 (i'-K)-EROs, show signs of active star formation (Fig. 2). An extended discussion is contained in Temporin et al. (2007b).

\section{A.3. High redshift objects in the K-selected spectroscopic sample: Effectiveness of the BzK Color selection}

We explored the effectiveness of the BzK diagnostic diagram (Daddi et al. 2004) in selecting high redshift galaxies from our K-selected spectroscopic sample. Our filter set was verified to be similar to the one used by Daddi et al. (2004), hence no color term was applied. Although the BzK method succeeds in identifying the high-redshift galaxies in our spectroscopic sample, we found a $64 \%$ contamination by low-redshift galaxies in the relevant area of the diagram, $\mathrm{BzK}=\left(\mathrm{z}^{\prime}-\mathrm{K}\right)-\left(\mathrm{B}-\mathrm{z}^{\prime}\right)>-0.2$, which is expected to be populated by star-forming galaxies at redshift $\mathrm{z}>1.4$ (Fig: 3). True high-redshift 

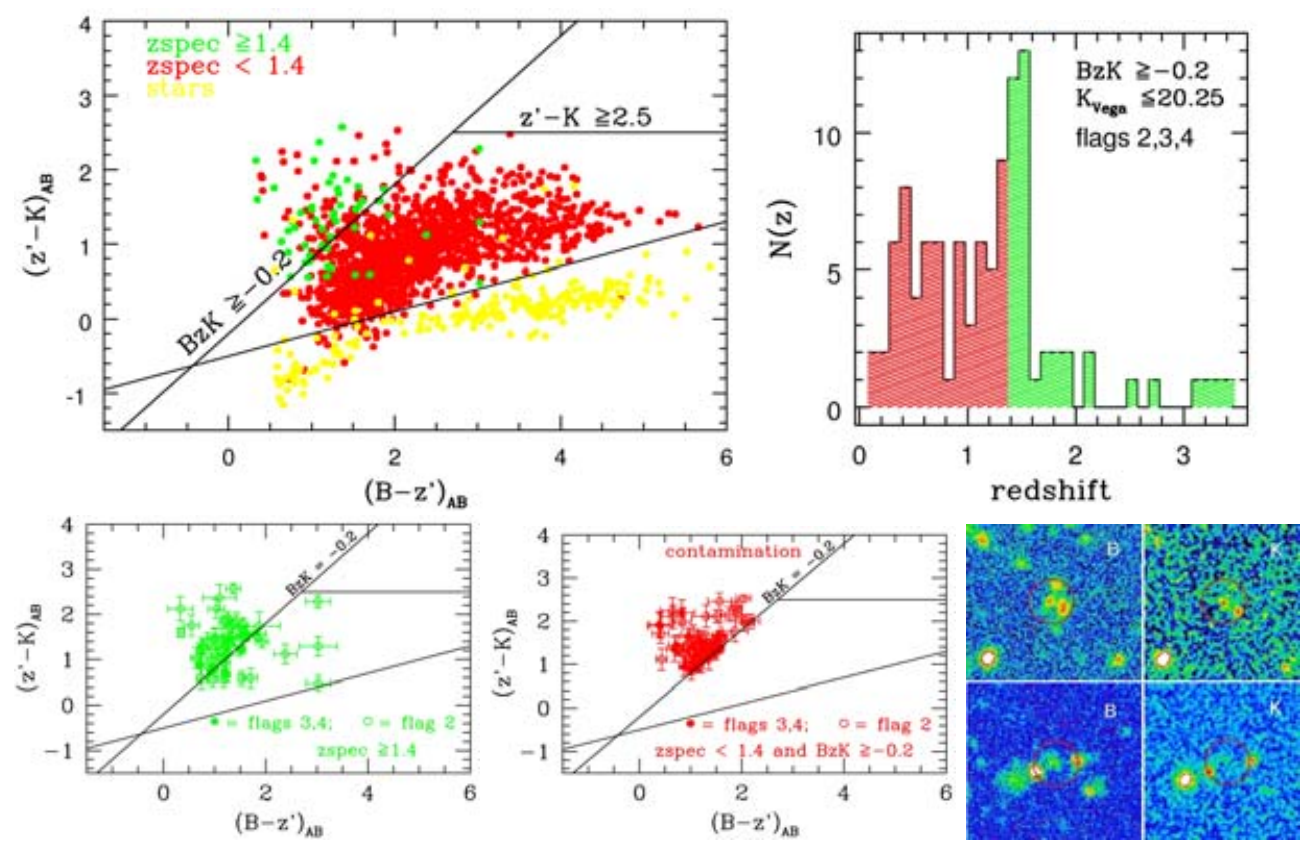

Figure 3. Upper left. BzK diagnostic diagram for the K-selected spectroscopic sample. Upper right. Redshift distribution of galaxies with $\mathrm{BzK} \geqslant-0.2$. Lower left BzK diagrams for galaxies with $\mathrm{z}>1.4$, irrespective of their colors (green), and for galaxies with $\mathrm{z}<1.4$ and $\mathrm{BzK} \geqslant-0.2$ (red). Lower right. Example of B and K-band images of low-redshift galaxies in multiple systems, which contaminate the high-redshift locus of the BzK diagram. The red circle has a radius of 3 arcsec.

galaxies and low-redshift contaminants are shown separately in Fig. 3. Error bars are from SExtractor (Bertin and Arnouts 1996) measurements. Apparently, photometric errors do not justify the observed contamination. Also, in all three bands involved, the magnitude distribution of the contaminant galaxies and that of high redshift galaxies are similar.

Interestingly, an inspection of the images of these objects revealed that $\sim 55 \%$ of the contaminants are members of tight (projected) pairs or multiple systems. Two such examples are shown in Fig. 3. The contamination rate lowers to $44 \%$ when tight pairs or multiple systems like those in Fig. 3, for which photometry is necessarily less reliable, even in our sub-arcsec seeing images, are discarded. We note that the $\mathrm{I}_{\mathrm{AB}}=24$ magnitude limit of the spectroscopic survey, which disfavours high-redshift and particularly red objects, might play a role in producing a higher contamination than usually observed (e.g. $\sim 20 \%$ in the spectroscopic sample of Daddi et al. 2004).

The observed SEDs of objects with redshift $>1.4$ and $\mathrm{BzK}>-0.2$ closely approaches Coleman, Wu, and Weedman (1980) templates of late-type galaxies, in agreement with the expectations for this color selection. The composite spectrum obtained for these high redshift sources (Fig. 4) actually shows the typical features of a late-type star forming galaxy.

\section{Acknowledgements}

This research has been developed within the framework of the VVDS consortium. This work has been partially supported by the CNRS-INSU and its Programme National de Cosmologie (France), and by Italian Ministry (MIUR) grants COFIN2000 (MM02037133) and COFIN2003 (no.2003020150). The VLT-VIMOS observations have been carried out 

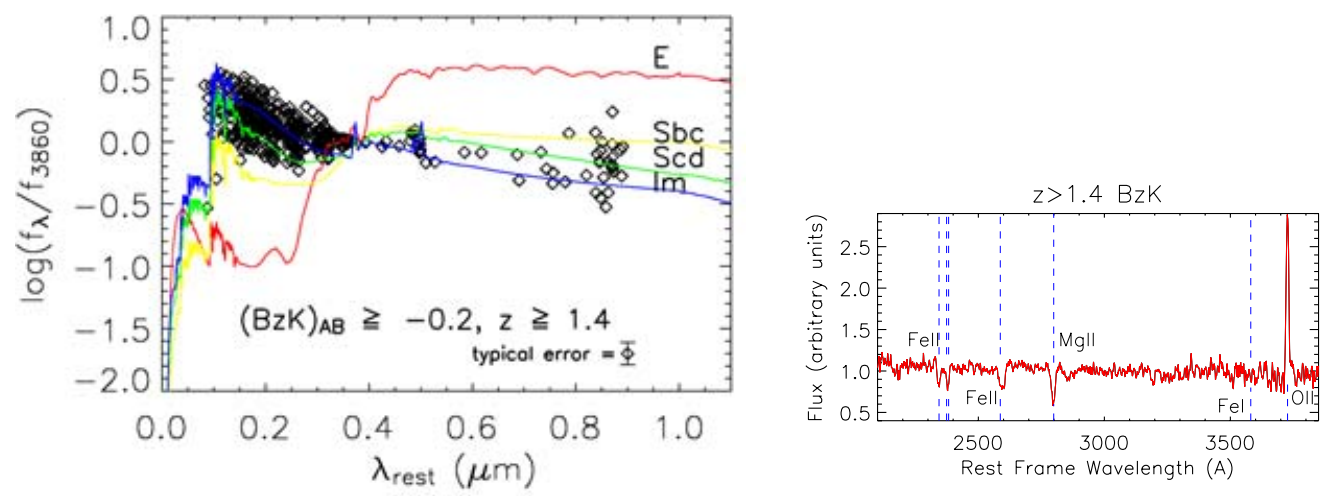

Figure 4. Left. Rest-frame observed SEDs of z $>1.4$ galaxies selected for having BzK $>-0.2$. Right. Composite spectrum of the same galaxies.

on guaranteed time (GTO) allocated by the European Southern Observatory (ESO) to the VIRMOS consortium, under a contractual agreement between the Centre National de la Recherche Scientifique of France, heading a consortium of French and Italian institutes, and ESO, to design, manufacture and test the VIMOS instrument.

\section{References}

Bertin, E. \& Arnouts, S. 1996 A $\mathscr{E} A S$ 117, 393

Coleman, G. D., Wu, C.-C., \& Weedman, D. W. 1980 ApJS 43, 393

Daddi, E., Cimatti, A., Renzini, A. et al. 2004, ApJ 617, 746

Ilbert, O., Arnouts, S., McCracken, H. J. et al. 2006, $A \mathscr{E} A$ 457, 841

Iovino, A., McCracken, H. J., Garilli, B. et al. 2005, A\&\&A 442, 423

Le Févre, O., Vettolani, G., Garilli, B. et al. 2005, A\&A 439, 845

McCracken, H. J., Radovich, M., Bertin, E. et al. 2003, A\&A 410, 17

Temporin, S., Iovino, A., McCracken, H. J., \& the VVDS Team 2007a, A\&A in preparation

Temporin, S., Iovino, A., McCracken, H. J., \& the VVDS Team $2007 \mathrm{~b}, A \mathscr{\xi} A$ in preparation

\section{Complete author list}

S. Temporin ${ }^{1}$, A. Iovino ${ }^{1}$, H. J. McCracken ${ }^{2,5}$, M. Bolzonella $^{3}$, M. Scodeggio $^{4}$, D. Bottini ${ }^{4}$, B. Garilli ${ }^{4}$, V. Le Brun ${ }^{6}$, O. Le Fèvre ${ }^{6}$, D. Maccagni ${ }^{4}$, J. P. Picat ${ }^{7}$, R. Scaramella ${ }^{8,10}$, L.

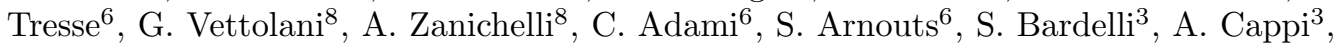
S. Charlot ${ }^{2,9}$, P. Ciliegi ${ }^{3}$, T. Contini ${ }^{7}$, O. Cucciati ${ }^{1,14}$, S. Foucaud ${ }^{21}$, P. Franzetti ${ }^{4}$, I. Gavignaud $^{11}$, L. Guzzo ${ }^{1}$, O. Ilbert ${ }^{20}$, B. Marano ${ }^{12}$, C. Marinoni ${ }^{18}$, A. Mazure ${ }^{6}$, B. Meneux ${ }^{1,4}$, R. Merighi ${ }^{3}$, S. Paltani ${ }^{15,16}$, R. Pellò ${ }^{7}$, A. Pollo ${ }^{6,17}$, L. Pozzetti ${ }^{3}$, M. Radovich $^{13}$, G. Zamorani ${ }^{3}$, E. Zucca ${ }^{3}$, M. Bondi ${ }^{8}$, A. Bongiorno ${ }^{12}$, J. Brinchmann ${ }^{19}$, S. de la Torre ${ }^{6}$, F. Lamareille ${ }^{3}$, Y. Mellier ${ }^{2,5}$, P. Merluzzi ${ }^{13}$, D. Vergani ${ }^{4}$, C. J. Walcher ${ }^{6}$

${ }^{1}$ INAF-Osservatorio Astronomico di Brera, Via Brera 28, 20121 Milano, Italy email: giovanna.temporin@brera.inaf.it

${ }^{2}$ Institut d'Astrophysique de Paris, UMR 7095, 98 bis Bvd Arago, 75014 Paris, France

${ }^{3}$ INAF-Osservatorio Astronomico di Bologna, Via Ranzani 1, Bologna, Italy

${ }^{4}$ INAF-IASF, Via Bassini 11, Milano, Italy

${ }^{5}$ Observatoire de Paris, LERMA, 61 Avenue de l'Observatoire, 75014 Paris, France

${ }^{6}$ Laboratoire d'Astrophysique de Marseille, UMR 6110 CNRS-Université de Provence, BP8, 13376 Marseille Cedex 12, France 
${ }^{7}$ Laboratoire d'Astrophysique de l'Observatoire Midi-Pyrénées (UMR 5572) - 14, avenue E. Belin, F31400 Toulouse, France

${ }^{8}$ IRA-INAF - Via Gobetti,101, I-40129, Bologna, Italy

${ }^{9}$ Max Planck Institut fur Astrophysik, 85741, Garching, Germany

${ }^{10}$ INAF-Osservatorio Astronomico di Roma - Via di Frascati 33, I-00040, Monte Porzio Catone, Italy

${ }^{11}$ Astrophysical Institute Potsdam, An der Sternwarte 16, D-14482 Potsdam, Germany ${ }^{12}$ Università di Bologna, Dipartimento di Astronomia - Via Ranzani,1, I-40127, Bologna, Italy

${ }^{13}$ INAF-Osservatorio Astronomico di Capodimonte - Via Moiariello 16, I-80131, Napoli, Italy

${ }^{14}$ Universitá di Milano-Bicocca, Dipartimento di Fisica - Piazza delle Scienze, 3, I-20126 Milano, Italy

${ }^{15}$ Integral Science Data Centre, ch. d'Écogia 16, CH-1290 Versoix

${ }^{16}$ Geneva Observatory, ch. des Maillettes 51, CH-1290 Sauverny, Switzerland

${ }^{17}$ Astronomical Observatory of the Jagiellonian University, ul Orla 171, 30-244 Kraków, Poland

${ }^{18}$ Centre de Physique Théorique, UMR 6207 CNRS-Université de Provence, F-13288 Marseille France

${ }^{19}$ Centro de Astrofsica da Universidade do Porto, Rua das Estrelas, 4150-762 Porto, Portugal

${ }^{20}$ Institute for Astronomy, 2680 Woodlawn Dr., University of Hawaii, Honolulu, Hawaii, 96822

${ }^{21}$ School of Physics \& Astronomy, University of Nottingham, University Park, Nottingham, NG72RD, UK 